Research Article

\title{
How Does the Heterogeneity of Members Affect the Evolution of Group Opinions?
}

\author{
An Lu $\mathbb{D}^{1},{ }^{1,2}$ Haifeng Ling, ${ }^{1,2}$ and Zhengping Ding ${ }^{1,2}$ \\ ${ }^{1}$ School of Management of Hefei University of Technology, Hefei 230009, Anhui, China \\ ${ }^{2}$ Key Laboratory of Process Optimization and Intelligent Decision Making, Ministry of Education, Hefei 230009, Anhui, China \\ Correspondence should be addressed to An Lu; luan76@hfut.edu.cn
}

Received 27 September 2020; Revised 3 December 2020; Accepted 19 December 2020; Published 4 January 2021

Academic Editor: Ruguo Fan

Copyright (C) 2021 An Lu et al. This is an open access article distributed under the Creative Commons Attribution License, which permits unrestricted use, distribution, and reproduction in any medium, provided the original work is properly cited.

Understanding the impact of heterogeneity on the evolution of group opinions can enlighten us on how to effectively organize, redesign, and improve decision-making efficiency. This article explores mainly the effects of heterogeneity on the evolution of group opinions. It is found that the heterogeneity of individuals' openness has an important influence on the ability to aggregate group opinions. According to the average amount of clusters and Herfindahl-Hirschman Index (HHI) under different network structures, heterogeneity often improves the ability. In addition, for the small-world network and random network, there is little difference in the aggregation ability from both the average amount of clusters and the Herfindahl-Hirschman Index. While for the regular network, the ability is obviously weaker than that of the other two. This result also shows that the randomness of interaction between members will enhance the cohesion of a group.

\section{Introduction}

Through a combination of theoretical and experimental work, it has become clear that many complex collective behavioral patterns can emerge via self-organizing processes from individuals using simple interaction rules [1-3]. However, individuals in groups are not all equal, and the phenotypic variation that is selectively maintained in populations results in individual heterogeneity within groups. A considerable amount of theoretical and empirical evidence shows that heterogeneity plays a fundamental role in collective behavior, structure, and function. For example, the prevalence of individuals' heterogeneity in animal behavior (or personality) $[4,5]$ suggests that it may play a fundamental role in driving the movement and function of animal groups [6, 7], including its collective decision-making, foraging performance, and predator avoidance. In the study of human group behavior, evidence of the influence of individual heterogeneity on group behavior is also found. For example, using evolutionary game models, Ma et al. [8] found that heterogeneous link weight can largely promote cooperation in spatial prisoner's dilemma. In the spatial public goods game, the heterogeneity or diversity of groups will not only increase the level of cooperation but also lead to the heterogeneity of wealth distribution $[9,10]$. Li et al. [11] illustrated the collapsing behavior of complex networks was induced by the individual heterogeneity based on a dynamic model. Iwanaga et al. [12] found that heterogeneous individuals may lead to unexpected collective behaviors and emergence.

The evolution of group opinions is an important research direction for collective behavior. However, there exist some subtle differences among the above topics. In this field, scholars mainly focus on the phenomenon of consensus, fragment, and polarization. Moreover, the opinion interaction rules between members are usually based on the psychological mechanism of compromise or conformity, which makes collective behavior be different from the above topics.

In order to explain the consensus, splitting, or polarization, scholars built various opinion models. Among these models, the Hegselmann and Krause (HK) model [13] is one of the most important continuous opinion models based on bounded confidence. Agents update their opinions 
repeatedly and simultaneously at discrete time steps by taking an average of the opinions "close enough" to their own. The model explains the split, polarization, or consensus of in reality. Scholars quickly paid attention to the model and found that the HK model has many special properties. It is proved that a consensus would be reached in finite time if and only if the opinion profile was an $\varepsilon$-chain at any time [14]. If trust between two agents is mutual, then every agent acquires a little bit of self-confidence, positive weights do not converge to zero, and the HK model will converge [15]. Francesca and Ceragioli et al. [16] proposed a continuoustime perspective evolution model similar to the previous ones based on kinetic methods.

The above studies are based mostly on homogeneous agents, but individuals in a group are often heterogeneous in reality. For example, some are willing to accept the opinions of others, while others are unwilling to accept the opinions of others. Thereafter, a large amount of literature researched the heterogeneous case. The first is the study of opinion leaders, which refers to an individual who has a greater influence on other individuals in terms of psychological conscious behaviors, such as viewpoints, attitudes, beliefs, and motivations in the process of people's opinion exchanges [17]. Many scholars have focused on public opinion leaders in the context of party elections and marketing activities, updated rules according to different opinions, constructed opinion dynamic models, and used computer simulation experiments to analyze the role of opinion leaders. For example, Watts and Dodds [18] used a series of computer simulation studies and found that in most cases, the huge influence belt is not driven by opinion leaders but rather by a group of key highly vulnerable individuals. The literature [19] divided the individuals into two categories, i.e., dominant individuals (public opinion leaders) and dependent individuals (ordinary individuals) based on the level of individuals in the social network. The results show that the existence of opinion leaders will significantly change the evolution and final state of the opinions of the entire social network group. Moreover, in a social network where the level of trust is heterogeneous, the strength of the leadership of opinion leaders is determined mainly by the social network. Individuals with a low level of trust and individuals at the subordinate level jointly determine the level of trust in opinion leaders. Other studies, such as [20-22], also discussed the evolution of opinions in the presence of opinion leaders in groups.

In addition, the literature [23-25] discussed the influence of stubborn individuals on the evolution of group opinions. For example, the literature [26, 27] discussed the role of some members of the group as "trust." Lorenz [15] proposed an improved model of continuous opinion evolution of trust parameters with heterogeneous trust parameters in reality when the trust thresholds of group members cannot be consistent. In this paper, those with smaller trust parameters are called close-minded individuals, while those with larger trust thresholds are called openminded individuals. It was found that even the trust thresholds of the two types of individuals were lower than the consistent critical threshold of the homogeneous model.
Literature [22] studied the evolution of opinion when members within the community have heterogeneous trust thresholds and used the approach of setting the trust threshold to three levels, from low to high. The first level represents conservative individuals, the second level represents general individuals, and the third level represents open individuals. The evolution of the group perspective was studied by setting the proportion of the three types of individuals in the group, and the results showed that the number of final opinion groups strongly depended on the proportion of these three groups of people. A consensus can be easily reached when most agents in a group are open. Meanwhile, when most agents in a group are conservative, reaching a consensus is not easy. The number of opinion clusters rises significantly when this proportion rises. In addition, this article also discusses the effects of group size on the evolution of opinion. Agents are divided into two types, conservative and open, based on the heterogeneity of agents, and the percentage of conservative individuals is observed at $0 \%, 10 \%, 20 \%$, and $30 \%$ [28]. A similar approach was also used in [29].

Although some studies have been conducted on the heterogeneous situation, as far as we know, the effects of heterogeneity still need to be further explored. Inspired by the existing work, in this paper, we focus on how the group's ability to aggregate opinions is affected by the distribution pattern under different network structures.

1.1. Contribution of This Paper. In this article, we broke through the limitation that the study of heterogeneity is only multilevel. Also, we built a group opinions evolution model based on heterogeneity and network structure. Furthermore, we used the indicator HHI to measure the ability to aggregate group opinions. Finally, we found that random interactions can enhance the ability. Last, we found the distribution of individual openness affects the aggregation ability of group opinions. For inverted triangle distribution (ITD), it is more conducive to the emergence of mainstream opinions, and the polarization phenomena will have less chance to occur.

This paper is organized as follows: In the second part, we proposed a heterogeneous model under the network structure environment. In the third part, we analyzed the evolution law of group opinion with a small-world network structure under the condition that the individual openness level follows a uniform distribution. We compared the triangular distribution, inverted triangular distribution, and one-point distribution under the group aggregation capability. In the fourth part, we summarized the conclusion and the limitations of this study and proposed future research direction.

\section{Model Formulation}

Let us consider a population with $n$ individuals under a certain network structure. The relationship between individuals is represented by $G=(V, E)$, where $V=\{1,2, \ldots, n\}$ is the vertex set and $E \subseteq V \times V$ is an edge set. If an individual $i$ is affected by $j$, it is represented by a directed edge $i \longrightarrow j$. 
In the original HK model, the interactions are global. In other words, the HK model is based on a complete graph. Considering the heterogeneity of individuals and the locality of interactions in reality, this paper improves the original model: We assume that interactions occur only between neighbors. Their relationship is reflected by the adjacency matrix $A=\left(a_{i j}\right)_{n \times n}$. Each individual $i$ has a bounded confidence threshold when accepting the opinion of $j$. Each individual has their level of individual openness. In this paper, we consider the heterogeneity of individuals and set this threshold for any individual $i$ as $l_{i}$.

Let the opinion of $i$ at time $t$ be $O_{i}(t)$. So heterogeneous opinion updating model can be written as follows:

$$
O_{i}(t+1)=\left|N_{i}(t)\right|^{-1} \sum_{j \in N_{i}(t)} O_{j}(t),
$$

where $N_{i}(t)=\left\{j \mid a_{i j} \neq 0\right.$ and $\left.\left|O_{i}(t)-O_{j}(t)\right| \leq l_{i}\right\}$ and denotes the number of neighbors of $i$.

In a homogeneous case, if there is a crack between two agents at time $t$, then there will be a crack at time $t+1$. Also, group opinions will keep order preservation (see $[12,30]$ ). But the two properties will no longer hold in our model. In some cases, due to the heterogeneity of individuals' openness, the interaction between individuals is unidirectional rather than symmetrical, which results in the complexity and asymmetry of opinion evolution (see Figure 1).

Remark: In the figure, the $X$-axis represents the time step, and the $Y$-axis represents the opinion values of all members in a group.

\section{How Does the Heterogeneity of Members Affect the Evolution of Group Opinions?}

3.1. Uniform Distribution. In contrast with [19], heterogeneous individuals were set as continuous random variables, wherein continuous random variables follow a special distribution. We explore whether the heterogeneity of individual openness affects the aggregation ability of group opinions by designing the research as follows. We embed individuals in small-world network (WS) and random network (ErdOs-Renyi) and assume that the level of openness of individuals follows a uniform distribution. We then observe the change of the average amount of clusters (AOC) when the uniform distribution parameters are varied. The average openness level is fixed to ensure comparability. We also set individuals different initial opinion values, which are distributed randomly between 0 and 1 .

In Figure 2 (panels (a), (b), and (c)), the leftmost point represents the uniform distribution $(0.01,0.39)$. In each panel, the point represents the group with the strongest heterogeneity. The point at the rightmost point represents a single point distribution with parameter 0.2 , which is a homogeneous case. In order to eliminate the influence of the initial opinions value of different individuals, we repeated the experiment 300 times to get the average amount of clusters (AOC). From each panel in Figure 2, we observed without exception that with the increase of the number of edges in the network, AOC decreases continuously. That is

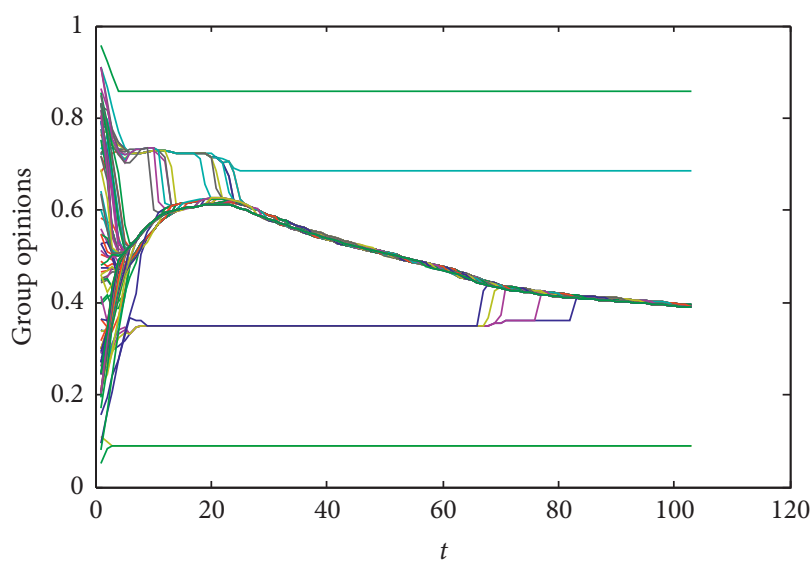

FIgURe 1: Asymmetry and complexity of the evolution in heterogeneous situations.

to say, the increase in interaction will lead to an increase in the ability to aggregate. We can see that whatever network structure is, the reduction of heterogeneity will lead to an increase of AOC, especially when the minimum level of openness is relatively high. In other words, when the average openness level of individuals is fixed, the stronger the heterogeneity, the stronger the ability of group opinions to aggregate, especially when all the group members have a certain level of openness. In addition, under different network structures, the AOC varies greatly. In the regular network, it is difficult to achieve consensus, and group opinions will split into many clusters. For random networks and small-world networks, the difference in the number of clusters is very small (about between 3 and 8). It indicates that the regular network structure is not conducive to the convergence of group opinions. This conclusion is similar to the conclusion of other group behaviors.

In Figure 2, the $X$-axis represents the lowest openness level of all individuals, and the $Y$-axis represents the average amount of clusters.

In order to further explore the influence of heterogeneity on the evolution of group views, we designed three different network structures, random network, WS-based smallworld network, and regular network. And suppose there are three possible heterogeneous populations: U-shaped distribution, inverted $U$-shaped distribution, and single point distribution. For the first two types of distribution, we use triangular distribution (TD) and inverted triangular distribution (ITD).

3.2. The Other Three Different Distributions. We assume that individual openness follows three different distributions: TD, ITD, and one-point distribution to explore the effect of the dynamic changes of individual openness and heterogeneity on the evolution, as shown in Figure 3.

Case 1. The openness level is between $\left[l_{1}, l_{2}\right]$ and follows $\mathrm{TD}$, where most people are moderate.

Case 2. The openness level is between $\left[l_{1}, l_{2}\right]$ and follows ITD, where most people are conservative or open-minded. 


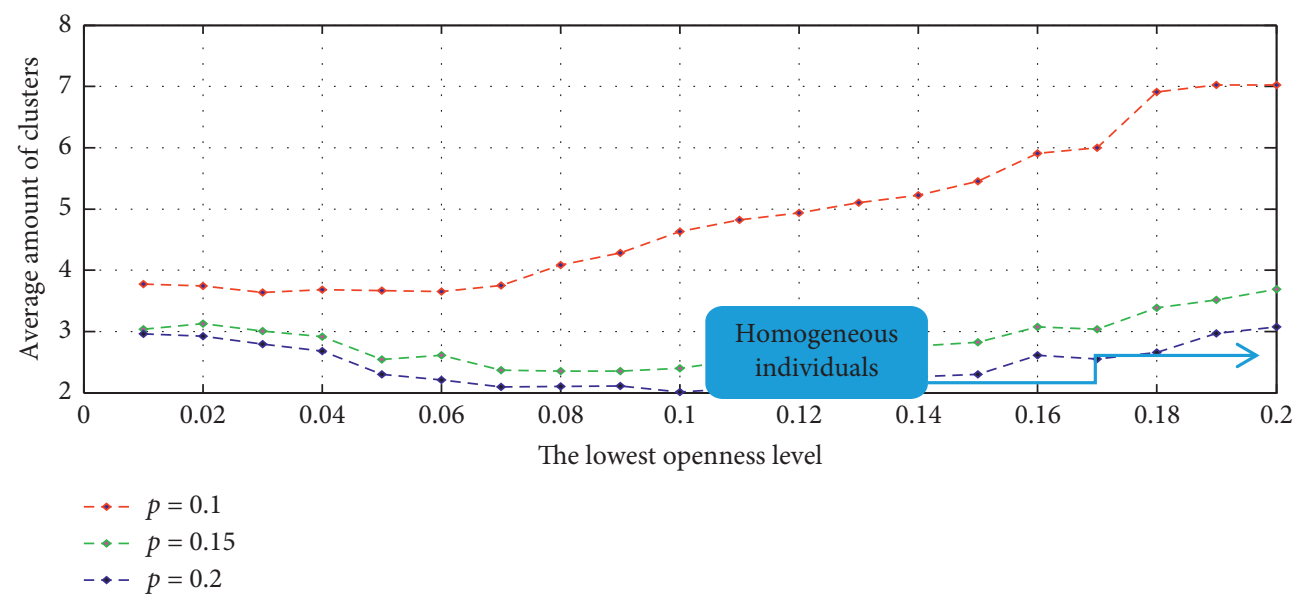

(a)

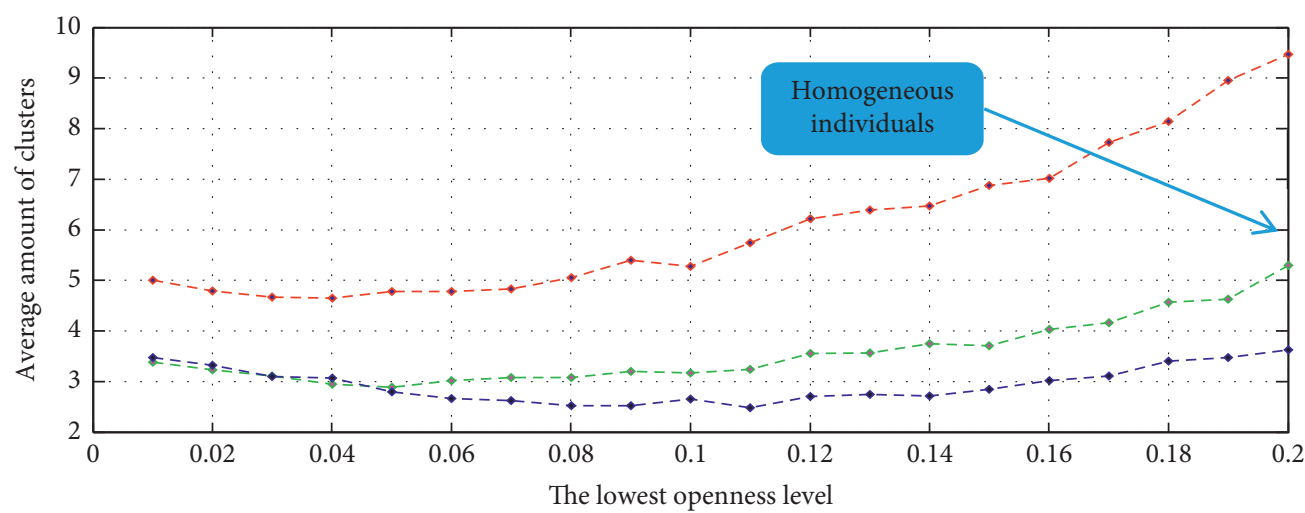

- - WS $(50,0.2)$

- WS $(50,0.4)$

- WS $(50,0.6)$

(b)

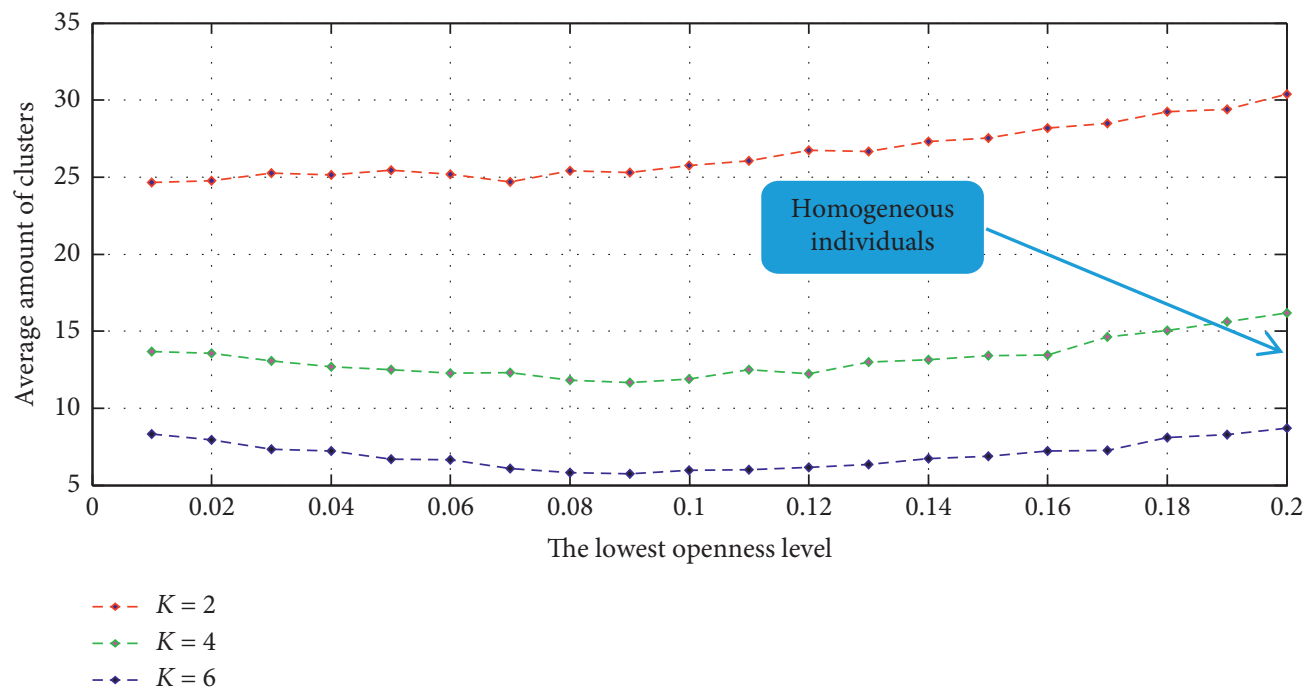

(c)

Figure 2: Influence of heterogeneity on AOC under different network densities.

Case 3. The openness levels are completely equal.

In Figure 3, the black line represents the triangle distribution (TD), where $X$-axis represents the openness level in a group, and $Y$-axis represents the density function or the proportion of the population at a given level. The red and blue lines represent ITD and homogeneous cases, 


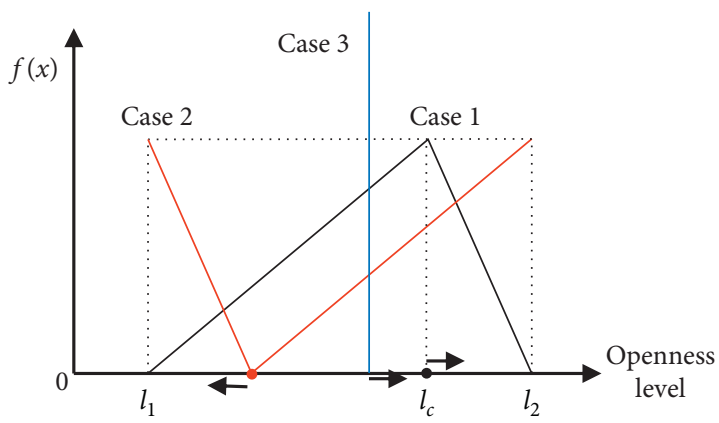

Figure 3: Probability density function of three cases.

respectively. Using the mean of TD as a benchmark, when $l_{c}$ moves to the right, the lowest point of Case 2 moves to the left and Case 3 moves to the right accordingly.

In Case 1, we can calculate the cumulative distribution function as follows:

$$
F_{1}(x)= \begin{cases}\frac{\left(x-l_{1}\right)^{2}}{\left(l_{2}-l_{1}\right)\left(l_{c}-l_{1}\right)}, & \left(l_{1} \leq x<l_{c}\right), \\ 1-\frac{\left(l_{2}-x\right)^{2}}{\left(l_{2}-l_{c}\right)\left(l_{2}-l_{1}\right)}, & \left(l_{c} \leq x<l_{2}\right),\end{cases}
$$

and

$$
x=F_{1}^{-1}(y)= \begin{cases}l_{1}+\sqrt{y\left(l_{2}-l_{1}\right)\left(l_{c}-l_{1}\right)}, \quad\left(y \leq \frac{l_{c}-l_{1}}{l_{2}-l_{1}}\right), \\ l_{2}-\sqrt{(1-y)\left(l_{2}-l_{c}\right)\left(l_{2}-l_{1}\right)}, & \left(y>\frac{l_{c}-l_{1}}{l_{2}-l_{1}}\right) .\end{cases}
$$

For Case 2,

$$
\begin{gathered}
F_{2}(x)= \begin{cases}\frac{\left(l_{c}-l_{1}\right)^{2}-\left(l_{c}-x\right)^{2}}{\left(l_{2}-l_{1}\right)\left(l_{c}-l_{1}\right)}, & l_{1} \leq x<l_{c}, \\
\frac{\left(l_{c}-l_{1}\right)\left(l_{2}-l_{c}\right)+\left(x-l_{c}\right)^{2}}{\left(l_{2}-l_{1}\right)\left(l_{2}-l_{c}\right)}, & l_{c} \leq x \leq l_{2},\end{cases} \\
x=F_{2}^{-1}(y)= \begin{cases}l_{c}-\sqrt{\left(l_{c}-l_{1}\right)^{2}-y\left(l_{2}-l_{1}\right)\left(l_{c}-l_{1}\right)}, & y \in\left[0, \frac{l_{c}-l_{1}}{l_{2}-l_{1}}\right], \\
l_{c}+\sqrt{y\left(l_{2}-l_{1}\right)\left(l_{2}-l_{c}\right)-\left(l_{c}-l_{1}\right)\left(l_{2}-l_{c}\right)}, & y \in\left[\frac{l_{c}-l_{1}}{l_{2}-l_{1}}, 1\right] .\end{cases}
\end{gathered}
$$

Using the discretization technique, the openness level of the $i$ th individual is set as $F_{1}^{-1}(i / N)$ in Case 1 and $F_{2}^{-1}(i / N)$ in Case 2, respectively.

We fix the left endpoint of TD and the right endpoint ( 0.1 and 0.38 , respectively). Using the mean of TD as a benchmark, $l_{c}$ increases from 0.1 to 0.39 . For comparability, the mean of the other two distributions is always equal to $\left(0.1+0.38+l_{c}\right) / 3$. More precisely, the lowest point of the inverted triangle is $0.1+0.38-l_{c}$, and the homogeneous case is that all individuals have the same openness level $\left(0.1+0.38+l_{c}\right) / 3$.

In Figure 4, the $Y$-axis represents the average amount of clusters (AOC) or Herfindahl-Hirschman Index (HHI). The $X$-axis represents the mode of triangle distribution, where the change of $l_{c}$ represents the change of the distribution pattern of the openness level in a group.

We observe the change of AOC in Figure 4. The red lines represent regular networks, while the green lines represent WS networks and the blue lines represent random networks. All the networks have the same number of edges. The diamond mark represents triangular distribution. The circular mark represents an inverted triangle distribution and the cross mark represents a homogeneous case.

Almost all curves in panel (a) have a negative slope, which indicates that the capability of aggregating will improve with the increase of average openness level. We also note that for a homogeneous case, the effect of the increase is considerably obvious. While for the heterogeneous case, the effect is relatively weak. To some extent, it indicates the importance of heterogeneity. When heterogeneity is sufficient, even if the average openness level gets lower or higher, it will not seriously affect the aggregating ability. Also, the ability under regular network is weaker than that of the other two network structures. For TD and ITD, there seems to be little difference. As shown in panel (a), under regular network and small-world network, the AOC of ITD is less than that of TD. But in random networks, the AOC of ITD is slightly more than that of the other two distributions.

In order to comprehensively understand the aggregation ability of these two distributions, we use the HHI index in economics to measure the ability of aggregating opinions. Specifically, HHI measures opinion concentration in the form of a sum of the squared "shares of all clusters" in the 


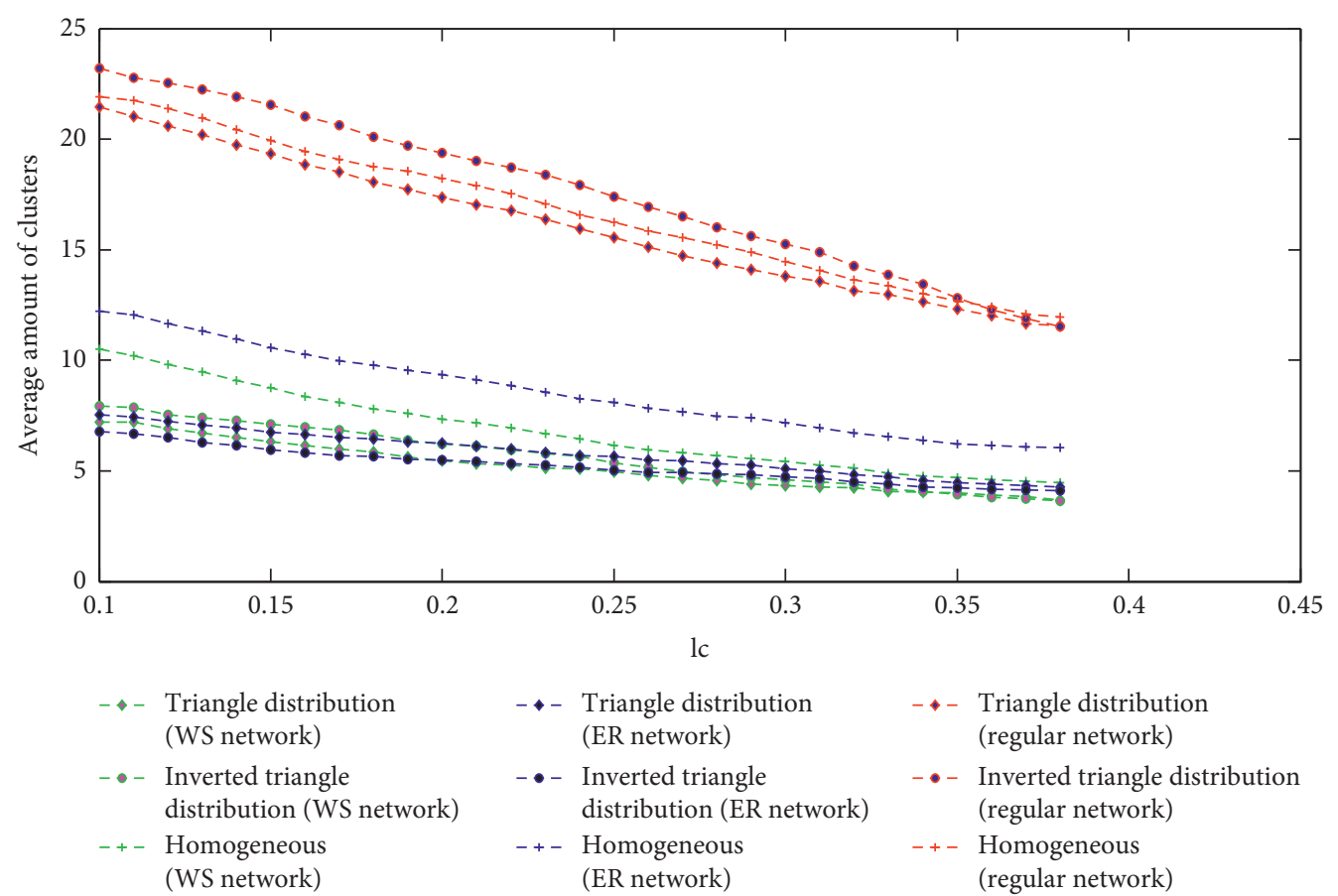

(a)

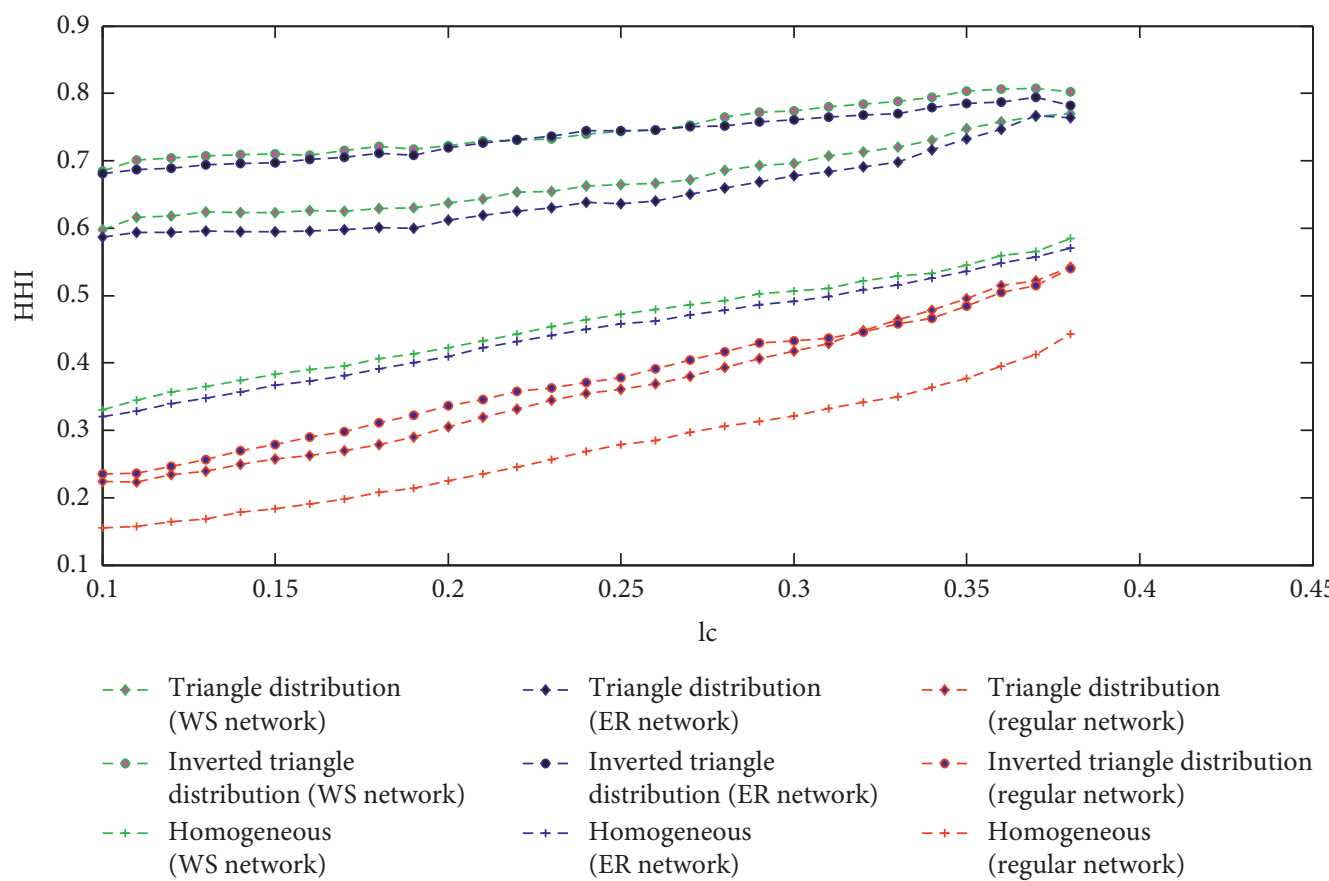

(b)

FIGURE 4: Influence of $l_{c}$ on the AOC and HHI under different network structures.

group, which means that it takes into account all the clusters [31]. For example, if the AOC is the same in two scenarios, but the largest clusters in the first scenario has more people, then the HHI index in the first scenario will be higher. This means that the first scenario has a higher ability to aggregate opinions.

Panel (b) in Figure 4 shows that with the continuous increase of $l_{c}$, HHI keeps increasing. This is a bit different from panel (a). In a small-world network, for example, TD has less AOC and lower HHI than ITD. The reason is that the size of each cluster in TD is relatively close. A cluster with a large size is not easy to appear, and as a result, it is easier to form the opinion polarization, so the HHI index will be lower.

Similarly, we fix the highest openness level and $l_{c}$, and change the lowest openness level from 0.01 to 0.19 , as shown in panel (a) and (b) in Figure 5. We fix the lowest openness level and $l_{c}$, and the highest openness level is changed from 


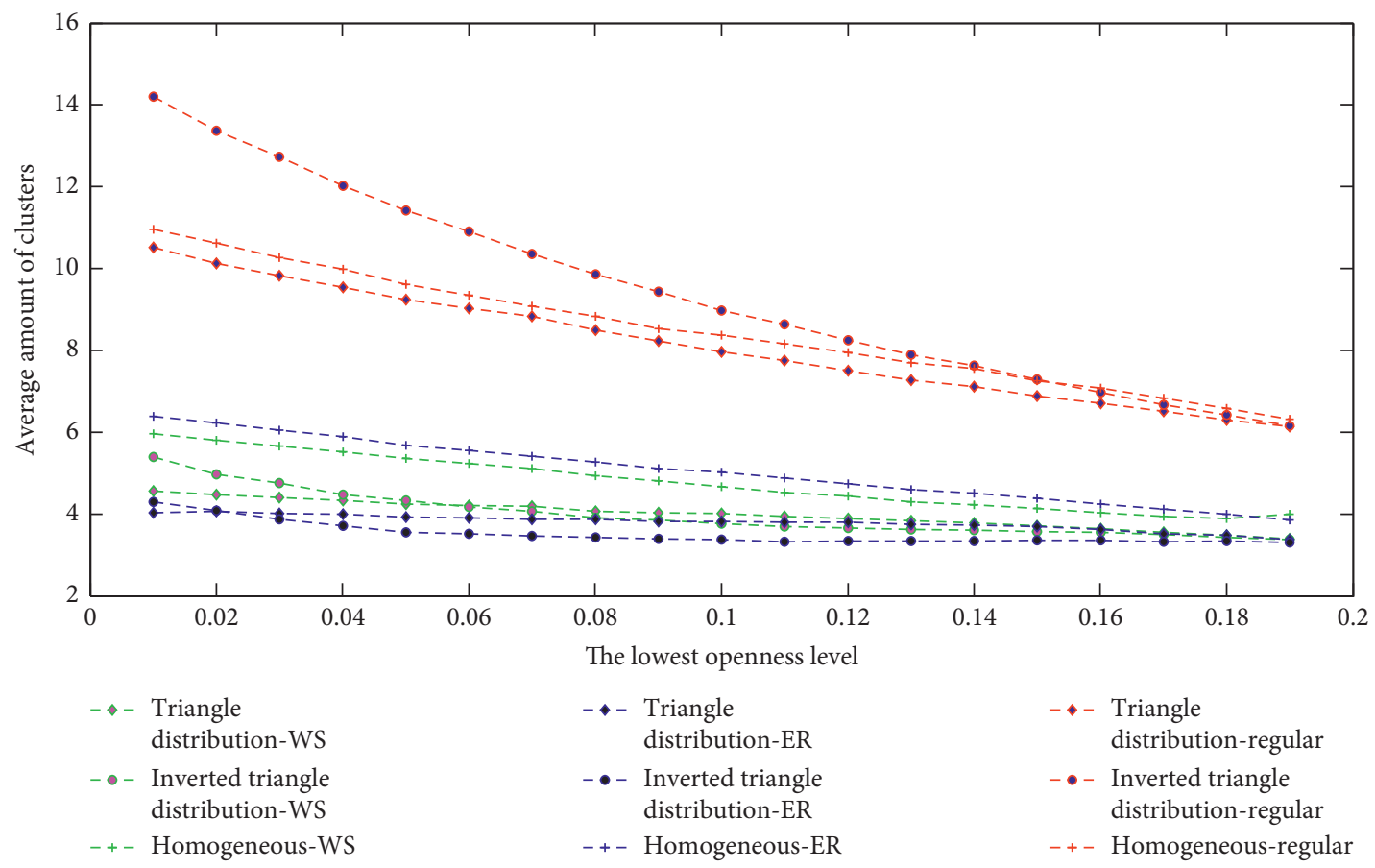

(a)

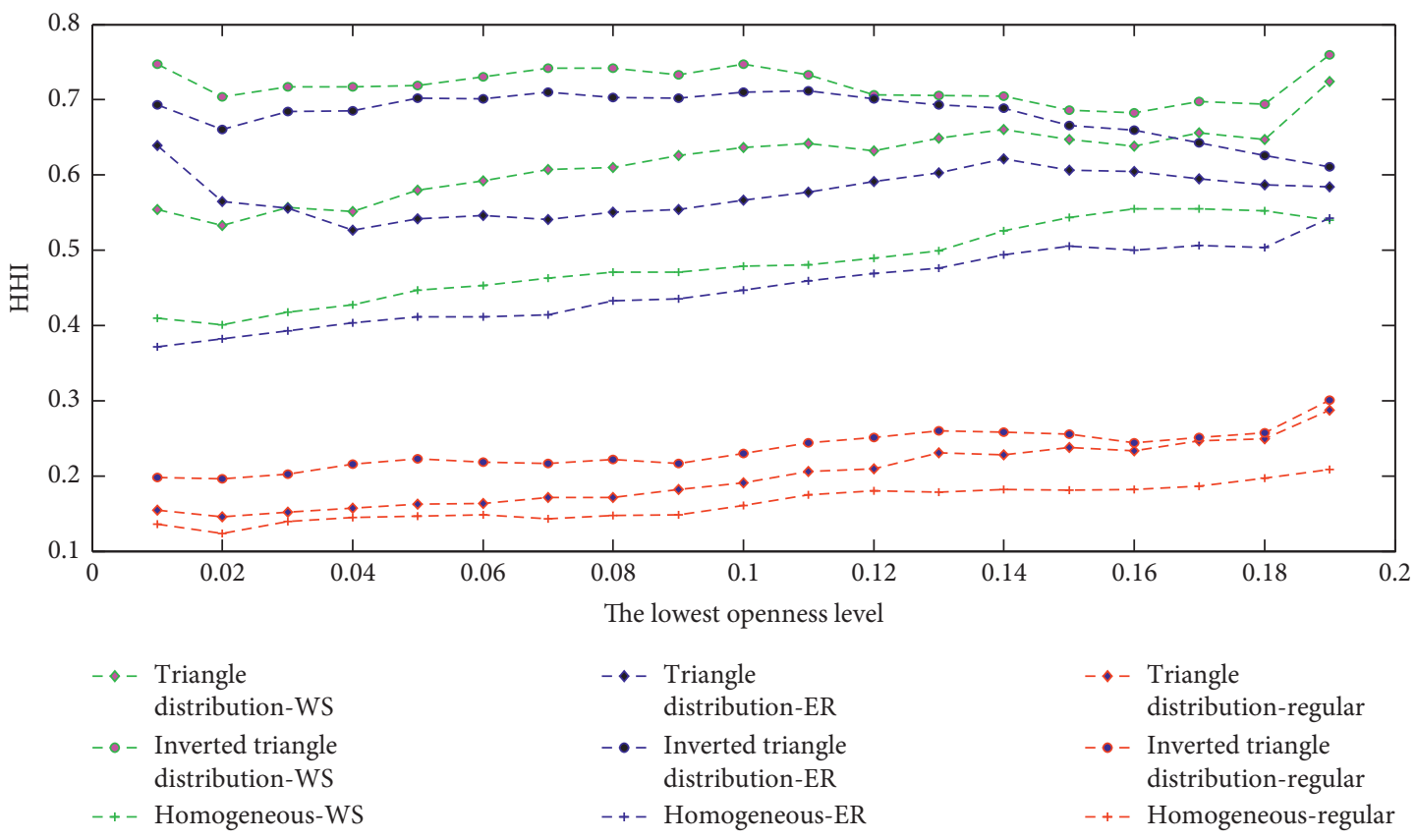

(b)

Figure 5: Continued. 


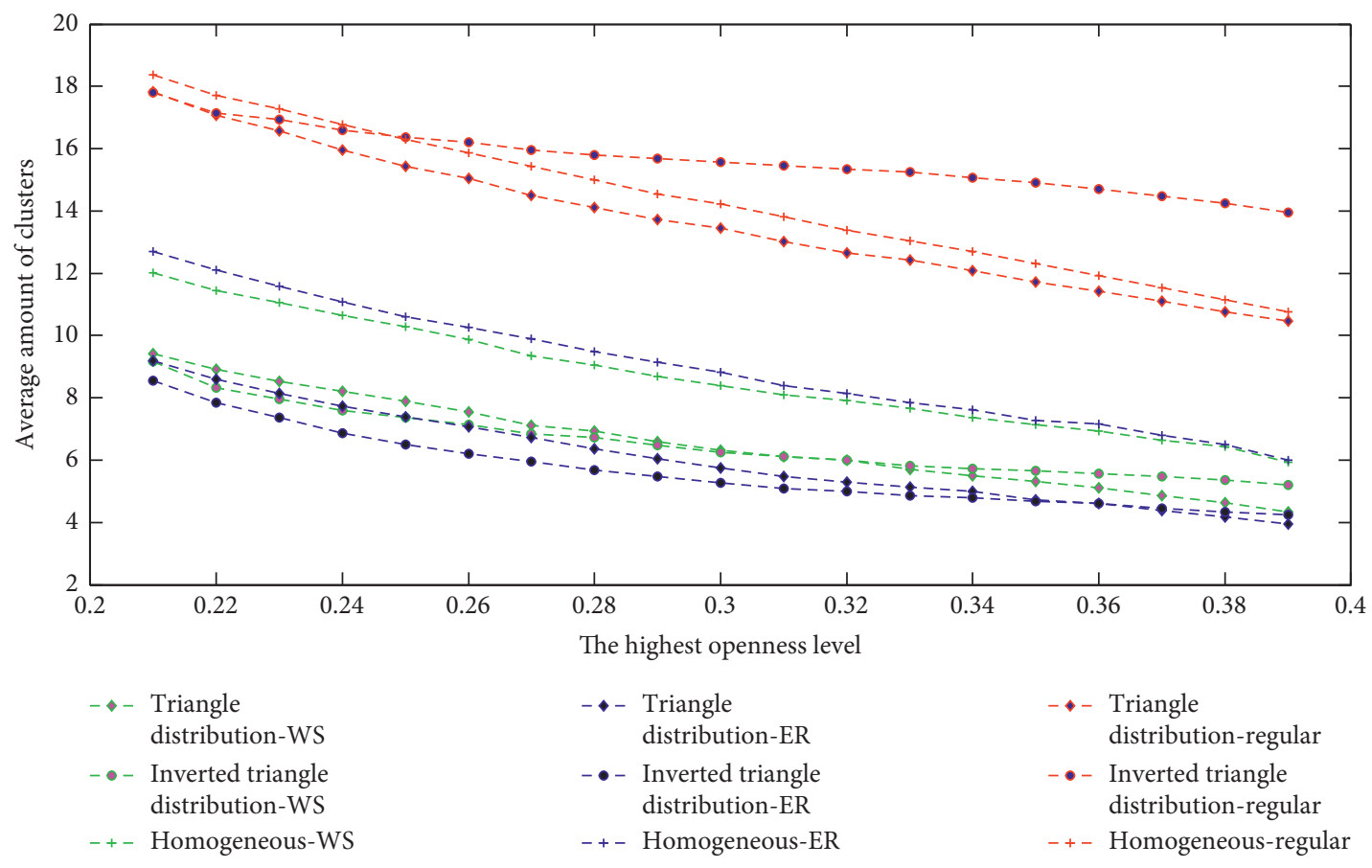

(c)

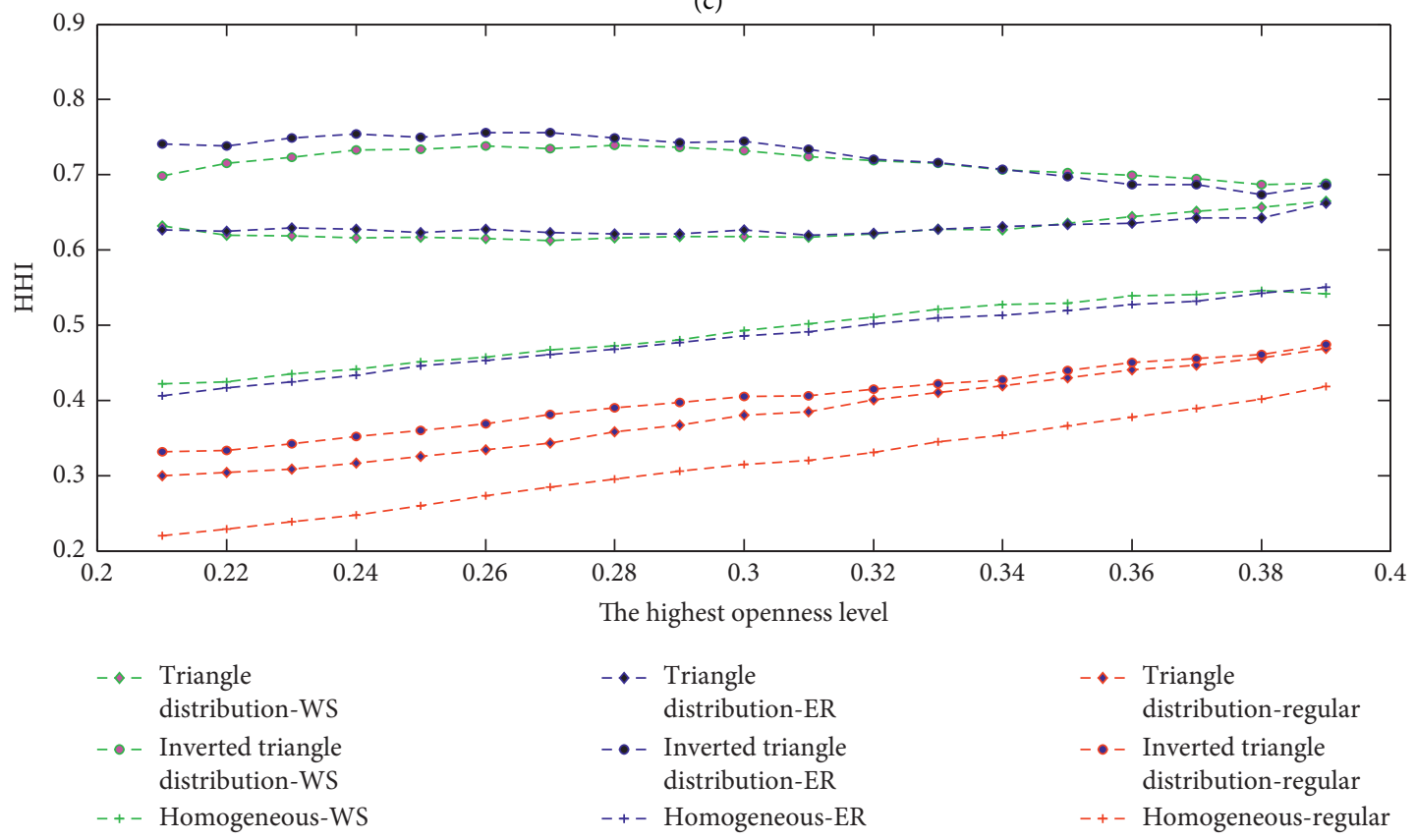

(d)

FIGURE 5: Influence of the lowest openness level or the highest openness level on the AOC and HHI.

0.21 to 0.39 . All the results are shown in panel (c) and (d) in Figure 5. We observe the changes of AOC and HHI and find the following:

(1) Whatever the network structure is, with the continuous improvement of the minimum level of openness, AOC keeps on decreasing, which shows that the average openness level is an important factor affecting the ability to aggregate group opinions (panel (a) in Figure 5).
(2) Whatever the network structure is, the aggregation ability of the homogeneous case is not as good as that of the heterogeneous one.

(3) The ability of aggregating various opinions is weak under a regular network, while that of a random network and small-world network is almost the same.

(4) The small-world network and the random network view group are almost the same in number, but the 
HHI under the small-world network structure is higher than that of the random network (panel (b) in Figure 5). The reason is that in the random network, there is little difference in the size of opinion clusters. It also shows that random networks are more difficult to form mainstream opinions than small-world networks.

(5) Compared with TD, the ITD is more prone to form large groups and mainstream opinion.

In Figure 5, the $Y$-axis represents the average amount of clusters (AOC) or Herfindahl-Hirschman Index (HHI). The $X$-axis represents the lowest openness level or the highest openness level of a group, where the change in the $X$-axis represents the change of the distribution pattern of the openness level in a group.

\section{Conclusion and Implication}

This paper finds that the heterogeneity of group members' openness has an important influence on the ability to aggregate group opinions. According to the AOC and $\mathrm{HHI}$, under different network structures, heterogeneity often improves the aggregation ability to aggregate group opinions. For small-world networks and random networks, there is little difference in the aggregation ability from both AOC and HHI. But for the regular network, the ability is obviously weaker than that of the other two. This result shows that the randomness of interaction between members will enhance the cohesion of a group.

The distribution of the openness of heterogeneous groups also affects the ability to aggregate group opinions. When the average openness level goes up, the aggregation ability is improved significantly. For inverted triangle distribution, it is more conducive to the emergence of mainstream opinion, and the polarization phenomena will have less chance to occur.

Understanding the impact of heterogeneity on the evolution of group opinions can enlighten us on how to effectively organize redesign and improve decision-making efficiency. If we want to enhance the cohesion of a group, members should be diverse in openness aspect. Also, the interaction should be as random as possible. For example, how do we arrange seats at round-table meetings? We should let open-minded and close-minded sit together so as to interact randomly enough, in which we can prevent the adverse impact of the regular network.

We use repeated experiments and Monte Carlo simulation to eliminate the influence of individual initial opinions on the evolutionary results. In fact, the initial opinion of an individual undoubtedly has a nonnegligible effect on the process of evolution. In the future, we will intend to study the interaction between individual heterogeneity and individual initial opinions, which is undoubtedly more challenging and meaningful.

\section{Data Availability}

The data used in this article are simulated, and the authors can provide the source code for the data generation if needed.

\section{Conflicts of Interest}

The authors declare that they have no conflicts of interest.

\section{Acknowledgments}

This work was supported by the Major Program of the National Natural Science Foundation of China (91846201), the Foundation for Innovative Research Groups of the National Natural Science Foundation of China (71521001), the National Natural Science Foundation of China (71722010, 91746302, and 71872060), and Research Fund for the Doctoral Program of Higher Education of China (JZ2019HGBZ0138).

\section{References}

[1] I. D. Couzin and J. Krause, "Self-organization and collective behavior in vertebrates," Advances in the Study of Behavior, vol. 32, no. 1, pp. 10-1016, 2003.

[2] D. J. T. Sumpter, Collective Animal behavior, Princeton University Press, William Street, Princeton, New Jersey, 2010.

[3] J. E. Herbert-Read, "Understanding how animal groups achieve coordinated movement," The Journal of Experimental Biology, vol. 219, no. 19, pp. 2971-2983, 2016.

[4] D. Réale, S. M. Reader, D. Sol, P. T. McDougall, and N. J. Dingemanse, "Integrating animal temperament within ecology and evolution," Biological Reviews, vol. 82, no. 2, pp. 291-318, 2007.

[5] A. M. Bell, S. J. Hankison, and K. L. Laskowski, "The repeatability of behaviour: a meta-analysis," Animal Behaviour, vol. 77, no. 4, pp. 771-783, 2009.

[6] M. M. Webster and A. J. W. Ward, "Personality and social context," Biological Reviews, vol. 86, no. 4, pp. 759-773, 2011.

[7] M. Wolf and J. Krause, "Why personality differences matter for social functioning and social structure," Trends in Ecology \& Evolution, vol. 29, no. 6, pp. 306-308, 2014.

[8] Z.-Q. Ma, C.-Y. Xia, S.-W. Sun, L. Wang, H.-B. Wang, and J. Wang, "Heterogeneous link weight promotes the cooperation in spatial prisoner's dilemma," International Journal of Modern Physics C, vol. 22, no. 11, pp. 1257-1268, 2011.

[9] J. Gao, Z. Li, T. Wu, and L. Wang, "Diversity of contribution promotes cooperation in public goods games," Physica A: Statistical Mechanics and Its Applications, vol. 389, no. 16, pp. 3166-3171, 2010.

[10] S. Meloni, C.-Y. Xia, and Y. Moreno, "Heterogeneous resource allocation can change social hierarchy in public goods games," Royal Society Open Science, vol. 4, no. 3, p. 170092, 2017.

[11] J. Li, J. Wang, S. Sun, and C. Xia, "Cascading crashes induced by the individual heterogeneity in complex networks," $A p$ plied Mathematics and Computation, vol. 323, pp. 182-192, 2018. 
[12] S. Iwanaga and A. Namatame, "Asymmetric coordination of heterogeneous agents," Ieice Transactions on Information and Systems, vol. 84, no. 8, pp. 937-944, 2001.

[13] R. Hegselmann and U. Krause, "Opinion dynamics and bounded confidence models, analysis, and simulation," Journal of Artificial Societies and Social Simulation, vol. 5, no. 3, 2002.

[14] J. C. Dittmer, "Consensus formation under bounded confidence," Nonlinear Analysis: Theory, Methods \& Applications, vol. 47, no. 7, pp. 4615-4621, 2001.

[15] J. Lorenz, "Heterogeneous bounds of confidence: meet, discuss and find consensus!" Complexity, vol. 15, no. 4, pp. 4352, 2010.

[16] F. Ceragioli and P. Frasca, "Continuous-time discontinuous equations in bounded confidence opinion dynamics," IFAC Proceedings Volumes, vol. 44, no. 1, pp. 1986-1990.1, 2010.

[17] S. Kodani and A. Sumitani, "The structure and dynamics of opinion-leader: from the mutsu-shi research in '81," Japanese Journalism Review Devoted to Studies in Journalism \& Mass Communication, vol. 32, pp. 5-24, 1983.

[18] D. J. Watts and P. S. Dodds, "Influentials, networks, and public opinion formation," Journal of Consumer Research, vol. 34, no. 4, pp. 441-458, 2007.

[19] Y. Zhao, Research on the Communication Mechanism of Public Opinion Based on Bounded Trust Rules in Sudden Mass Incidents, University of Electronic Science and Technology of China, Chengdu, Sichuan, China, 2014.

[20] N. Boccara, "Models of opinion formation: influence of opinion leaders," International Journal of Modern Physics C, vol. 19, no. 01, pp. 93-109, 2008.

[21] Y. Dong, Z. Ding, L. Martínez, and F. Herrera, "Managing consensus based on leadership in opinion dynamics," Information Sciences, vol. 397-398, pp. 187-205, 2017.

[22] Y. Zhao and G. Kou, "Bounded confidence-based opinion formation for opinion leaders and opinion followers on social networks," Studies in Informatics \& Control, vol. 23, no. 2, pp. 153-162, 2014.

[23] J. Ghaderi and R. Srikant, Opinion Dynamics in Social Networks with Stubborn Agents, Pergamon Press, Oxford, UK, 2014.

[24] A. Mukhopadhyay, R. R. Mazumdar, and R. Roy, "Majority rule based opinion dynamics with biased and stubborn agents," Acm Sigmetrics Performance Evaluation Review, vol. 44, no. 1, pp. 385-386, 2016.

[25] L. Stella, F. Bagagiolo, D. Bauso et al., "Opinion dynamics and stubbornness through mean-field games," in Proceedings of the Decision and Control, pp. 2519-2524, Los Angeles, CA, USA, December 2014.

[26] K. Fan and W. Pedrycz, "Opinion evolution influenced by informed agents," Physica A: Statistical Mechanics and Its Applications, vol. 462, pp. 431-441, 2016.

[27] M. Meadows and D. Cliff, The Relative Disagreement Model of Opinion Dynamics: Where Do Extremists Come From?, Springer Berlin Heidelberg, NY, New York, USA, 2016.

[28] B. M. Horio and J. R. Shedd, "Agent-based exploration of the political influence of community leaders on population opinion dynamics," in Proceedings of the Winter Simulation Conference, Las Vegas Nevada, NV, USA, December 2017.

[29] M. Pineda and G. M. Buendía, "Mass media and heterogeneous bounds of confidence in continuous opinion dynamics," Physica A Statistical Mechanics \& Its Applications, vol. 420, no. 1, pp. 73-84, 2014.

[30] F. Ceragioli and P. Frasca, "Continuous and discontinuous opinion dynamics with bounded confidence," Nonlinear
Analysis: Real World Applications, vol. 13, no. 3, pp. 12391251, 2012.

[31] I. Pavic, F. Galetic, and D. Piplica, "Similarities and differences between the CR and HHI as an indicator of market concentration and market power," British Journal of Economics, Management \& Trade, vol. 13, no. 1, pp. 1-8, 2016. 